\title{
Plasma 8-Isoprostane Is Increased in Preterm Infants Who Develop Bronchopulmonary Dysplasia or Periventricular Leukomalacia
}

\author{
TERHI AHOLA, VINETA FELLMAN, INGEMAR KJELLMER, KARI O. RAIVIO, AND \\ RISTO LAPATTO \\ Hospital for Children and Adolescents [T.A., V.F., K.O.R., R.L.], Helsinki University Central Hospital, \\ FIN-00029 Helsinki, Finland; Department of Pediatrics [I.K.], Göteborg University, S-41685 Gothenburg, \\ Sweden; and Department of Pediatrics [V.F.], Lund University, S-22185 Lund, Sweden
}

\section{ABSTRACT}

Our aim was to assess the plasma free 8-epi-prostaglandin $\mathrm{F}_{2 \alpha}$ (8-isoprostane) and ascorbyl radical as risk indicators for oxidative damage in extremely low birth weight infants (ELBWIs) and the effect of N-acetylcysteine (NAC) on these markers. Plasma samples were collected on days 3 and 7 of life from infants who were enrolled in a randomized, controlled trial in which i.v. NAC or placebo was administered to ELBWIs during the first week of life, with the aim of preventing bronchopulmonary dysplasia (BPD). Plasma 8-isoprostane was analyzed in 83 infants using an enzyme immunoassay kit. Ascorbyl radical concentration was measured in 61 infants with electron spin resonance spectroscopy. The 8 -isoprostane concentrations were similar in the NAC and placebo groups. In infants who later developed BPD or died $(n=29)$, the median (range) 8-isoprostane concentration was significantly higher $(p=0.001)$ on day 3 and day $7[50.0 \mathrm{pg} / \mathrm{mL}(19-360)$ and $57.0 \mathrm{pg} / \mathrm{mL}(14-460)$, respectively] than in survivors without BPD $[n=54 ; 34.5 \mathrm{pg} / \mathrm{mL}$ $(5-240)$ and $39.5 \mathrm{pg} / \mathrm{mL}(7-400)$, respectively]. The 8 -isoprostane levels increased significantly more $(p<0.05)$ in infants who later developed periventricular leukomalacia. NAC treatment or the later development of BPD was not related to the ascorbyl radical levels. The ascorbyl radical level decreased significantly in all groups from day 3 to day 7, but the difference between the groups was not significant. The mean (SD) ascorbyl radical level on day 3 was significantly higher $(p<0.01)$ in infants who later developed periventricular leukomalacia [287 (124) versus 194 (90)]. These data suggest that plasma 8-isoprostane could serve as a marker in assessing the risk for BPD development in ELBWIs. (Pediatr Res 56: 88-93, 2004)
Abbreviations
8-isoprostane, 8-epi-prostaglandin $\mathrm{F}_{2 \alpha}$ BPD, bronchopulmonary dysplasia
ELBWI, extremely low birth weight infant
IVH, intraventricular hemorrhage
MDA, malondialdehyde
NAC, N-acetylcysteine
NEC, necrotizing enterocolitis
PVL, periventricular leukomalacia
ROP, retinopathy of prematurity
ROS, reactive oxygen species

Free radical generation and oxidative injury have been implicated as causal factors in several complications of prematurity, such as bronchopulmonary dysplasia (BPD), retinopathy of prematurity (ROP), necrotizing enterocolitis (NEC), intraventricular hemorrhage (IVH), and periventricular leukomalacia (PVL) (1). Despite improved perinatal care, BPD remains one of the most important causes of long-term morbidity after preterm birth (2). Oxygen therapy and inflammatory neutrophil

Received August 6, 2003; accepted January 20, 2004.

Correspondence: Terhi Ahola, M.D., Hospital for Children and Adolescents, Research Laboratory, Biomedicum, 4th floor, P.O. Box 700, 00029 Helsinki, Finland; e-mail: terhi.ahola@helsinki.fi

Supported by Helsinki University Central Hospital (E.V.O.), Finnish Pediatric Research Foundation, and Päivikki and Sakari Sohlberg Foundation.

DOI: 10.1203/01.PDR.0000130478.05324.9D activation are considered important in increasing the production of reactive oxygen species (ROS) and the release of proinflammatory cytokines resulting in abnormal lung development. Evidence for increased oxidant production and oxidant-induced macromolecular damage has been demonstrated during the first week of life in infants who develop BPD $(2,3)$. Increased levels of volatile lipid peroxidation products have been shown to correlate with poor respiratory outcome and death in very low birth weight infants (4).

Preterm newborn infants are considered vulnerable to oxidative stress because of deficient antioxidant capacity. Even though the enzymatic antioxidant defenses of preterm infants seem relatively well developed, with the exception of catalase (5), the other antioxidant mechanisms are less well developed. Preterm infants have relative glutathione deficiency, inversely 
correlated with gestational age (6), and also lower plasma cysteine levels when compared with term infants (7). Furthermore, low levels of selenium, a component of glutathione peroxidases, have been shown in preterm infants (8). Glutathione acts both as a cosubstrate in peroxidase reactions and as a direct scavenger of reactive oxygen species.

Because oxidative stress is considered important in the pathogenesis of the complications of prematurity, several trials of antioxidant supplementation have been performed to improve the outcome of preterm infants (9-12). However, no or only minor beneficial effects on the clinical outcome have been achieved so far. N-acetylcysteine (NAC) is a precursor of cysteine and by itself a free radical scavenger (13). It has been shown to enter the cell and to be deacetylated to cysteine (14), which can replete intracellular glutathione. In adults with adult respiratory distress syndrome, NAC treatment reduced lung injury in some studies $(15,16)$ but not in others $(17)$. We carried out a randomized, multicenter, controlled trial to test the hypothesis that NAC administration during the first week of life would decrease the incidence of BPD in extremely low birth weight infants (ELBWIs). However, no difference was found in the incidence of BPD or death in the NAC-treated infants $(n=194)$ compared with placebo-treated infants $(n=197)$ (18).

Despite increasing indirect evidence of the role of oxidative injury in preterm infants, no clinically applicable specific indicator of oxidative stress is available for defining risk group infants or the effect of antioxidant therapy. F2-isoprostanes are a series of prostaglandin F2-like compounds produced in vivo independent of cyclo-oxygenase, as products of the radical-catalyzed lipid peroxidation. Measurement of F2-isoprostane concentrations has proved to be valuable in assessing oxidative stress in vivo, because they are specific products of lipid peroxidation. They are also stable compounds, present in all normal biologic fluids in detectable quantities, and they have been shown to increase in animal models of oxidant injury (19). Of the F2-isoprostanes, 8-epiprostaglandin $\mathrm{F}_{2 \alpha}$ (8-isoprostane) is one of the most abundant in humans. In term infants with severe respiratory failure, increased concentration of 8-isoprostane in tracheal aspirates has been found during the first $2 \mathrm{~d}$ of life (20).

An alternative way to demonstrate formation of free radicals is to measure stable radicals by means of spin trap analysis. When ascorbic acid, also an effective antioxidant, or its anion ascorbate reacts with a radical as a reducing agent, the transfer of one electron results in the formation of semi-dehydroascorbate radical (ascorbyl radical), a relatively stable radical with a biologic half-life of 30-60 min but stable at freezing temperature. It can be demonstrated and quantified in the tissue or plasma because of its characteristic electron spin resonance spectrum. The aim of the present study was to assess plasma 8-isoprostane and ascorbyl radical as indicators for the development of BPD or other ROS-related complications of prematurity in ELBWIs and the potential effects of i.v. NAC on these markers of oxidative stress.

\section{METHODS}

Subjects. Preterm infants who had a birth weight from 500 to $999 \mathrm{~g}$ and needed ventilatory assistance, either ventilator or nasal continuous positive airway pressure, were enrolled in a randomized, controlled trial in 10 Nordic academic intensive care units. The infants received i.v. NAC $\left(16-32 \mathrm{mg} \cdot \mathrm{kg}^{-1}\right.$. $\mathrm{d}^{-1}$, starting before the age of $36 \mathrm{~h}$ ) or placebo for $6 \mathrm{~d}$. The plasma samples for 8-isoprostane measurements reported here were obtained from 83 infants of the group $(n=123)$ enrolled in the Hospital for Children and Adolescents, University of Helsinki, Finland. Infants with adequate samples on days 3 and 7 were included. Ascorbyl radical was measured in 61 infants. The Research Ethics Committee of the hospital and the National Agency for Medicines in Finland approved the study. Written informed parental consent was obtained for each infant before trial enrollment.

The baseline characteristics of the study group infants did not differ from the other infants of the center or from the total study population ( $n=391)$, with the exception of intrauterine growth retardation; $46 \%$ of infants in the study group were small for gestational age compared with $38 \%$ in the entire trial study population. Maternal infection was defined as need of antibiotic treatment just before or during delivery because of clinical symptoms of infection, such as fever, or elevated C-reactive protein and/or premature rupture of membranes. BPD was defined as a need for supplemental oxygen or ventilatory assistance, either ventilator or nasal continuous positive airway pressure, at the age of $36 \mathrm{wk}$ gestation according to a recent proposal (2). The diagnoses of IVH and PVL were established by ultrasound examination of the brain, performed at least once during the first week and at the age of $28 \mathrm{~d}$. The main characteristics and the outcome were similar in NAC and placebo groups, with the exception of maternal infection. Five infants died, three of severe respiratory failure. In two infants, the lung disease was moderate, and the causes of death were pseudomembranous colitis and chylothorax as a result of vena cava thrombosis, respectively. Because all of the deceased infants probably would have fulfilled the criteria of BPD if they would have survived to the age of 36 wk gestation, we included them in the adverse outcome group and considered "BPD or death" as one entity in this study as well as in the trial. We made the analyses both with and without these infants. Excluding these infants from the analyses did not change the results significantly. When classified according to the main outcome BPD or death at the age of $36 \mathrm{wk}$ gestation versus no BPD, the baseline characteristics were similar with the exception of birth weight; infants who survived without BPD weighed significantly more $(p<0.05$; Table 1$)$. There were no differences in the baseline characteristics in infants with or without PVL. The incidence of maternal infection and postnatal sepsis was similar in both groups.

Sampling. Arterial blood samples $(1.0 \mathrm{~mL})$ were obtained from an indwelling catheter into heparin-coated test tubes on days 3 and 7 of life. These days were chosen to ensure that plasma NAC level remained stable during the study. Plasma was separated within $15 \mathrm{~min}$ and stored frozen at $-70^{\circ} \mathrm{C}$ until analyzed.

Determination of 8-isoprostane. Plasma free 8-isoprostane concentration was measured without further manipulation with a commercial enzyme immunoassay kit (Cayman Chemical, Ann Arbor, MI, U.S.A.). The samples were assayed in a 
Table 1. Baseline characteristics and outcome variables of the infants who later developed BPD or died compared with infants who survived without BPD

\begin{tabular}{|c|c|c|}
\hline Characteristic & $\begin{array}{l}\text { BPD or died } \\
\quad(n=29)\end{array}$ & $\begin{array}{l}\text { No BPD } \\
(n=54)\end{array}$ \\
\hline Birth weight [g (SD)] & $718 \quad(117)$ & $782(136) p<0.05$ \\
\hline Gestational age [wk (SD)] & $26.4(2.1)$ & $26.9(1.7)$ \\
\hline $\begin{array}{l}\text { Antenatal glucocorticoid therapy } \\
{[n(\%)]}\end{array}$ & $25 \quad(86)$ & $50 \quad(93)$ \\
\hline Preeclampsia $[n(\%)]$ & $(28)$ & $20 \quad(37)$ \\
\hline Maternal infection $[n(\%)]$ & $(45)$ & $13(24) p=0.052$ \\
\hline Male gender $[n(\%)]$ & $(55)$ & $21 \quad(39)$ \\
\hline $\begin{array}{l}\text { Small for gestational age }[<-2 \mathrm{SD} \text {; } \\
\quad n(\%)]\end{array}$ & $(38)$ & $27 \quad(50)$ \\
\hline $\begin{array}{l}\text { Respiratory distress syndrome } \\
{[n(\%)]}\end{array}$ & $23 \quad(79)$ & $48 \quad(89)$ \\
\hline Surfactant treatment $[n(\%)]$ & $24 \quad(83)$ & (85) \\
\hline NAC treatment $[n(\%)]$ & $(52)$ & (48) \\
\hline NAC starting age $[\mathrm{h}(\mathrm{SD})]$ & (7) & (10) \\
\hline Death $[n(\%)]$ & (17) & (0) \\
\hline Treated PDA $[n(\%)]$ & $(76)$ & (77) \\
\hline IVH grades $3-4[n(\%)]$ & (17) & (9) \\
\hline $\operatorname{PVL}[n(\%)]$ & $(10)$ & (9) \\
\hline ROP grade 3 or more $[n(\%)]$ & $5 \quad(17)$ & (9) \\
\hline NEC (operated) $[n(\%)]$ & $(10)$ & (9) \\
\hline $\begin{array}{l}\text { Sepsis (positive blood culture) } \\
\quad[n(\%)]\end{array}$ & (41) & $18 \quad(33)$ \\
\hline
\end{tabular}

Data are presented as means (SD) or numbers (\%). PDA, patent ductus arteriosus.

96-well plate coated with mouse anti-rabbit IgG MAb to 8 -isoprostane. An 8-isoprostane tracer bound to acetylcholinesterase was used to compete for binding sites. Samples were assayed at $50 \mu \mathrm{L}$ and read at $420 \mathrm{~nm}$ in a 96-well microplate reader (Multiscan EX; Thermo Labsystems Oy, Vantaa, Finland). The range of the standard curve was from 3.9 to 500 $\mathrm{pg} / \mathrm{mL}$.

Determination of ascorbyl radical. The intensity of the ascorbyl radical signal in $0.2 \mathrm{~mL}$ of plasma was measured with a Bruker ECS 106 electron spin resonance spectrometer. Spectrometer settings were as follows: field center, 3478.5 Gauss; modulation amplitude, 1.0 Gauss; microwave power, $10 \mathrm{~mW}$; microwave frequency, $9.74 \mathrm{GHz}$; scan range, 10 Gauss; scan rate, 60 Gauss $/ \mathrm{min}$; time constant, $0.02 \mathrm{~s}$. The intensity of the signal is displayed as arbitrary units and is proportional to the amount of radicals in the sample.

Statistical analysis. Data were analyzed using the SPSS 10.0 statistical package. The results of isoprostane analyses are given as medians and ranges, and the results of ascorbyl radical analyses are given as means and SDs. Differences in isoprostane and ascorbyl radical levels between the groups were determined using general linear model ANOVA for repeated measures. For achieving normal distribution, the 8-isoprostane data were $\log$ transformed before statistical analysis. Baseline data for the treatment groups were compared using the $t$ test for continuous variables or the Mann-Whitney $U$ test, as appropriate. Categorical baseline characteristics and outcomes were analyzed using the $\chi^{2}$ test. General linear model ANOVA for repeated measures were used to correct for baseline differences between the groups. All statistical tests were two-tailed. Statistical significance was set at $p<0.05$.

\section{RESULTS}

Free 8-isoprostane measurements. The median (range) 8 -isoprostane concentrations on day 3 and on day 7 were 37.0 $\mathrm{pg} / \mathrm{mL}(19-360 \mathrm{pg} / \mathrm{mL})$ and $44.0 \mathrm{pg} / \mathrm{mL}(7-460 \mathrm{pg} / \mathrm{mL})$ in the NAC group and $42.5 \mathrm{pg} / \mathrm{mL}(5-300 \mathrm{pg} / \mathrm{mL})$ and $44.5 \mathrm{pg} / \mathrm{mL}$ $(8-400 \mathrm{pg} / \mathrm{mL})$ in the placebo group. There was no significant difference between the treatment groups. In infants who later developed BPD or died $(n=29)$, the median 8 -isoprostane concentration was significantly higher on day 3 and day 7 [50.0 $\mathrm{pg} / \mathrm{mL}(19-360 \mathrm{pg} / \mathrm{mL})$ and $57.0 \mathrm{pg} / \mathrm{mL}(14-460 \mathrm{pg} / \mathrm{mL})$, respectively] when compared with infants who did not develop BPD $[n=54 ; 34.5 \mathrm{pg} / \mathrm{mL}(5-240 \mathrm{pg} / \mathrm{mL})$ and $39.5 \mathrm{pg} / \mathrm{mL}$ (7-400 pg/mL), respectively; Fig. 1] Although plasma 8-isoprostane levels correlated with birth weight, this difference was still significant when corrected for birth weight $(p=0.005)$. 8 -Isoprostane levels were highest in infants with severe BPD and correlated with the level of oxygen requirement at the age of 7 and $14 \mathrm{~d}$ but not at $28 \mathrm{~d}$.

In infants who later developed PVL $(n=8)$, the median 8 -isoprostane concentration was similar on day $3[39 \mathrm{pg} / \mathrm{mL}$ $(5-360 \mathrm{pg} / \mathrm{mL})$ versus $39.5 \mathrm{pg} / \mathrm{mL}(30-240 \mathrm{pg} / \mathrm{mL})]$ but was significantly increased on day 7 , when compared with infants who did not develop PVL [40 pg/mL (7-460 pg/mL) versus 80 $\mathrm{pg} / \mathrm{mL}$ (45-400 pg/mL), respectively; Fig. 2] Also the 8-isoprostane concentrations in infants who had severe (grade 3-4) IVH $[67.5 \mathrm{pg} / \mathrm{mL}(29-190 \mathrm{pg} / \mathrm{mL})$ and $81 \mathrm{pg} / \mathrm{mL}(18-490$ $\mathrm{pg} / \mathrm{mL}$ ) on day 3 and 7, respectively; $n=10)$ differed significantly from those without IVH $[36 \mathrm{pg} / \mathrm{mL}(5-360 \mathrm{pg} / \mathrm{mL})$ and $44 \mathrm{pg} / \mathrm{mL}(7-360 \mathrm{pg} / \mathrm{mL})$ on day 3 and 7 , respectively; $p<$ $0.01)$. The 8 -isoprostane concentrations were similar in infants with or without NEC or ROP. There were no differences in the 8 -isoprostane levels in infants with or without postnatal sepsis (verified by positive blood culture).

Ascorbyl radical signal. The mean (SD) ascorbyl radical level in arbitrary units on day 3 and on day 7 were 222 (108) and $136(54)$ in the NAC group $(n=29)$, and $190(87)$ and 126 (52) in the placebo group $(n=32)$, respectively. There was a

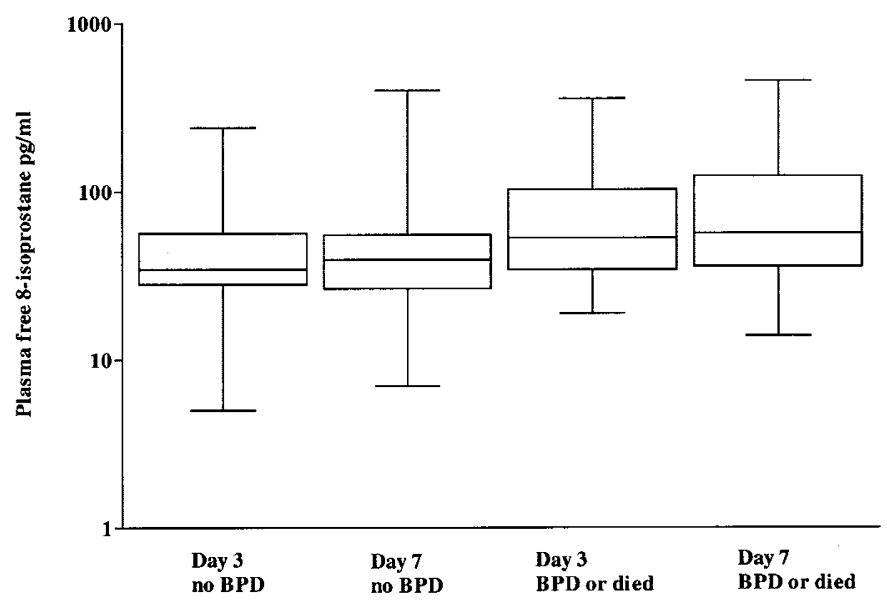

Figure 1. Plasma free 8-isoprostane levels on day 3 and day 7 of life were significantly higher in ELBWIs who later developed BPD or died $(n=29)$ when compared with ELBWIs who survived without BPD $(n=54)$. General linear model for repeated measures $p=0.005$. Values are shown in median levels (25th/75th box, total range). 


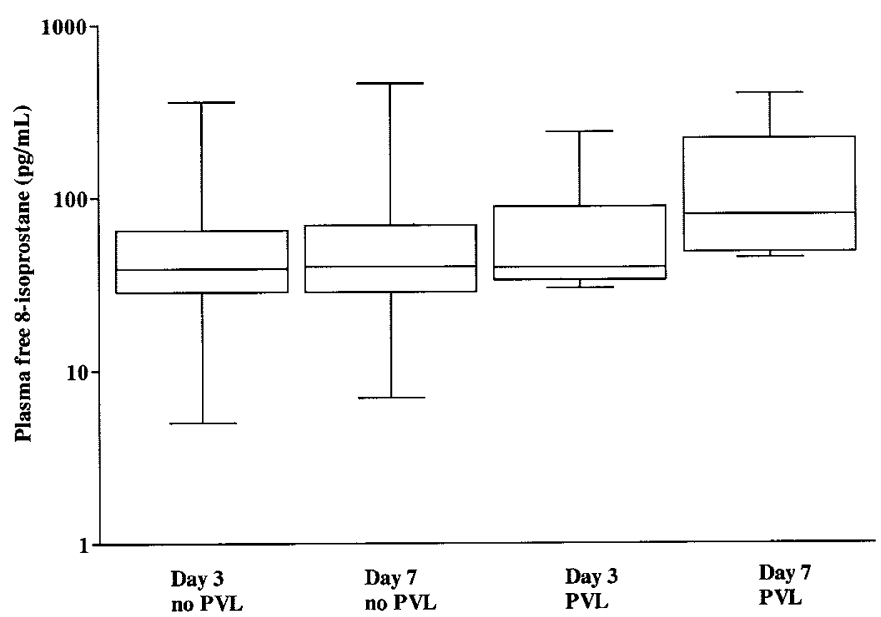

Figure 2. Plasma free 8-isoprostane levels were similar on day 3 but increased significantly in ELBWIs who later developed PVL $(n=8)$ compared with those without PVL $(n=75)$. General linear model for repeated measures $p<0.05$. Values are shown in median levels $(25$ th/ 75 th box, total range).

significant, similar decrease in both groups. The mean (SD) ascorbyl radical level was similar in infants who later developed BPD or died $(n=25)$ and in those without BPD $[n=36$; 218 (112) versus 196 (87) on day 3, and 139 (54) versus 125 (52) on day 7].

In infants who later developed PVL, the ascorbyl radical level was significantly higher on day 3 [287 (124) versus 194 (90); $p<0.01]$ but not on day 7 [127 (68) versus 131 (51); Fig. 3] The ascorbyl radical levels were similar in infants with or without severe IVH, NEC, or ROP. The ascorbyl radical levels did not correlate with the isoprostane levels.

\section{DISCUSSION}

Our study demonstrated that the level of plasma free 8-isoprostane is elevated during the first week of life in infants who develop BPD and PVL. The ascorbyl radical level was in-

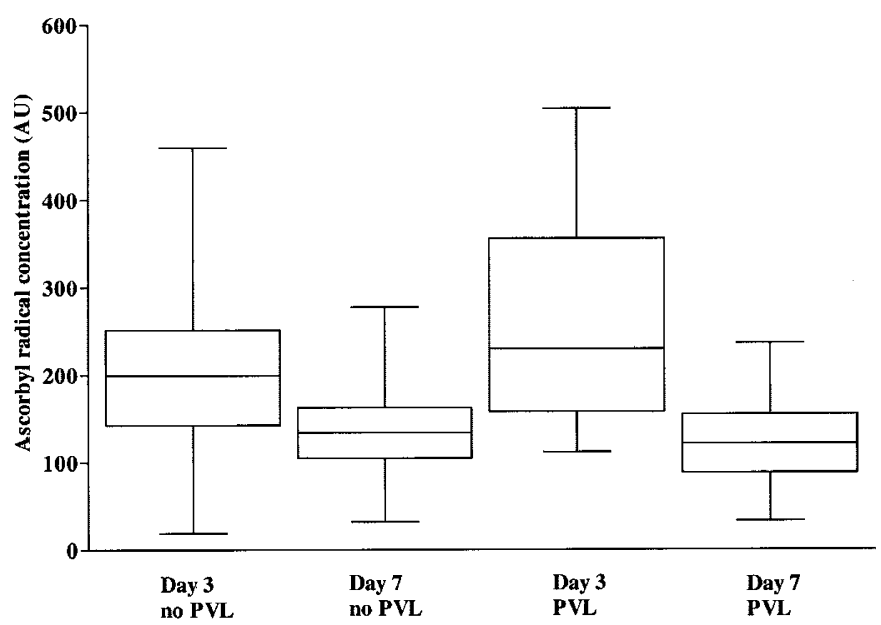

Figure 3. Plasma ascorbyl radical levels were significantly higher on day 3 of life in ELBWIs who later developed PVL $(n=7)$ when compared with infants without PVL $(n=54)$. General linear model for repeated measures $p<0.01$. The ascorbyl radical levels decreased from day 3 to day 7 significantly in both groups. Values are shown in median levels ( 25 th/75th box, total range). creased on day 3 in infants who later developed PVL. NAC treatment did not have any effect on the 8 -isoprostane or ascorbyl radical level.

It is possible to measure free plasma isoprostanes and total plasma isoprostanes after hydrolysis from phospholipids. Less than half of total plasma 8-isoprostane is present in free form (21). Free and bound plasma isoprostanes do not necessarily behave similarly, and it has been shown, e.g. in preeclamptic women, that plasma free but not total isoprostane levels are increased before delivery (22). Ideally, one would measure total lung tissue isoprostanes, but, this being impossible, we decided to measure free plasma isoprostanes to minimize sample manipulation that could lead to artifact formation. Sample purification with resins is possible, but there are problems with quantification, because binding to resins is never complete or specific. This would have had a large effect on our tiny samples. There are other, more specific methods to determine isoprostane concentration, such as gas chromatography/ mass spectrometry, which, however, was not available to us. Hence, we assumed that our method would create a large spread of values but no systematic error between the groups that would require careful interpretation. Nevertheless, our studies with unpurified plasma gave consistent results when measured repeatedly.

Elevated 8-isoprostane concentrations have been measured as a marker of in vivo oxidative stress during the first $2 \mathrm{~d}$ of life in tracheal aspirates of term infants with severe pulmonary disease (20), in bronchoalveolar lavage fluid in adults with interstitial lung diseases (23), in plasma in cystic fibrosis (24), and in exhaled breath condensate in adult respiratory distress syndrome (25). It has also been speculated that isoprostanes likely not only are markers of oxidative stress but also play a role in pulmonary pathophysiology, as they evoke important biologic responses in many cell types of the lung. 8-Isoprostane is a potent vasoconstrictor of pulmonary vascular and airway smooth muscle (26).

Earlier studies have shown evidence of increased markers of oxidative stress already during the first $2 \mathrm{~d}$ of life $(4,27,28)$. Unfortunately, in this study, plasma samples could not be obtained before receiving parental informed consent and enrollment in the trial, and tracheal fluid samples were not available. Whether the increase of 8-isoprostane in tracheal aspirates is associated with increased plasma 8-isoprostane levels has not been reported previously. We chose to study plasma 8-isoprostane and ascorbyl radical, which might also reflect the role of ROS in extrapulmonary complications of prematurity. To further clarify the role of the studied markers for assessing ROS-related pulmonary damage, one would need to study both tracheal aspirate and plasma samples repeatedly during the early postnatal phase. However, the present study suggests that oxidative stress is an important feature in the pathogenesis of BPD (4,27-29). Nycyk et al. (30) failed to show a correlation between BPD and lipid peroxidation, but they were able to show that elevated exhaled pentane levels are associated with the other complications of prematurity and may contribute to their severity. Direct evidence of increased production of ROS is difficult to get because of their short lifetime. Oxidative injury, however, can be evaluated, e.g. by measure- 
ment of markers of lipid peroxidation, protein carboxylation, and DNA base modification. The specificity and sensitivity of these markers is often criticized, and some of the analyses are difficult to perform and therefore are not practical in clinical use. In the study of Winterbourn et al. (31), the concentrations of plasma malondialdehyde (MDA) did not correlate with the respiratory outcome or with ROP. The thiobarbituric acidMDA assay, even when improved by HPLC separation, has been criticized as inaccurate, because the sample heating may create artificial aldehydes (32).

In the development of white matter injury, ischemia and reperfusion, as well as inflammation, seem to be important factors in the production of ROS (33). In a well-established neonatal rat model of hypoxic-ischemic brain injury, the production of ascorbyl radicals measured from brain homogenate amplified considerably during the early stage of reoxygenation (34). Increased concentration of F2-isoprostanes and formation of ascorbyl radicals in cerebrospinal fluid occur in traumatic brain injury, where ROS are considered important in pathogenesis (35). In the study of Inder et al. (36), the concentration of 8-isoprostane and MDA in cerebrospinal fluid was significantly higher in very low birth weight infants with white matter injury when compared with adult control subjects. They also showed a significant difference in the 8-isoprostane level between infants with cystic PVL in comparison with infants with a less severe form of white matter injury.

In our study, we showed significant differences in the plasma free 8-isoprostane concentrations in infants with or without PVL and severe IVH. Also, the plasma ascorbyl radical level was higher on day 3 in infants who later developed PVL. Our results support the hypothesis of free radical-mediated injury being a final common pathway to oligodendroglial cell death in cerebral white matter injury in preterm infants (33). However, the number of infants who developed PVL in our study was low, and further studies are needed to evaluate whether these markers could be valuable in assessing the risk of PVL in ELBWIs. It is possible that the elevation of the concentration of the ascorbyl radical marks the quenching of the radical cascade by ascorbic acid rather than potential pathologic mechanisms of white matter injury.

We failed to show any beneficial effects on the pulmonary outcome at the age of $36 \mathrm{wk}$ gestation with the supplementation of NAC as an antioxidant and a precursor of glutathione synthesis. The treatment with NAC did not have any influence either on the plasma free 8-isoprostane or on plasma ascorbyl radical levels. It has been speculated that the oxidative injury begins already before delivery or at birth, when the change of oxygen environment is the largest. Thus, the antioxidant treatment should possibly be started even earlier than in our study, with a mean starting age of $24 \mathrm{~h}$ postnatally.

In conclusion, the present study shows an association between elevated plasma free 8-isoprostane, BPD, and PVL. The spread of the values, however, was large, and further studies are needed to evaluate the usefulness of isoprostane measurements. Also, the ascorbyl radical signal in plasma was elevated in the early life of infants who later developed PVL.

\section{REFERENCES}

1. Saugstad OD 1998 Oxygen radical disease in neonatology. Semin Neonatol 3:229 238

2. Jobe AH, Bancalari E 2001 Bronchopulmonary dysplasia. Am J Respir Crit Care Med 163:1723-1729

3. Welty SE, Smith CV 2001 Rationale for antioxidant therapy in premature infants to prevent bronchopulmonary dysplasia. Nutr Rev 59:10-17

4. Pitkänen OM, Hallman M, Andersson SM 1990 Correlation of free oxygen radicalinduced lipid peroxidation with outcome in very low birth weight infants. J Pediatr 116:760-764

5. Asikainen TM, Raivio KO, Saksela M, Kinnula VL 1998 Expression and developmental profile of antioxidant enzymes in human lung and liver. Am J Respir Cell Mol Biol 19:942-949

6. Jain A, Mehta T, Auld PA, Rodrigues J, Ward RF, Schwartz MK, Martensson J 1995 Glutathione metabolism in newborns: evidence for glutathione deficiency in plasma, bronchoalveolar lavage fluid, and lymphocytes in prematures. Pediatr Pulmonol 20:160-166

7. Vina J, Vento M, Garcia Sala F, Puertes IR, Gasco E, Sastre J, Asensi M, Pallardo FV $1995 \mathrm{~L}$-cysteine and glutathione metabolism are impaired in premature infants due to cystathionase deficiency. Am J Clin Nutr 61:1067-1069

8. Darlow BA, Inder TE, Graham PJ, Sluis KB, Malpas TJ, Taylor BJ, Winterbourn CC 1995 The relationship of selenium status to respiratory outcome in the very low birth weight infant. Pediatrics 96:314-319

9. Darlow BA, Winterbourn CC, Inder TE, Graham PJ, Harding JE, Weston PJ, Austin NC, Elder DE, Mogridge N, Buss IH, Sluis KB 2000 The effect of selenium supplementation on outcome in very low birth weight infants: a randomized controlled trial. The New Zealand Neonatal Study Group. J Pediatr 136:473-480

10. Darlow BA, Graham PJ 2002 Vitamin A supplementation for preventing morbidity and mortality in very low birthweight infants. Cochrane Database Syst Rev (4):CD000501

11. Watts JL, Milner R, Zipursky A, Paes B, Ling E, Gill G, Fletcher B, Rand C 1991 Failure of supplementation with vitamin $\mathrm{E}$ to prevent bronchopulmonary dysplasia in infants less than 1,500 g birth weight. Eur Respir J 4:188-190

12. Davis JM, Rosenfeld WN, Richter SE, Parad MR, Gewolb IH, Spitzer AR, Carlo WA, Couser RJ, Price A, Flaster E, Kassem N, Edwards L, Tierney J, Horowitz S 1997 Safety and pharmacokinetics of multiple doses of recombinant human CuZn superoxide dismutase administered intratracheally to premature neonates with respiratory distress syndrome. Pediatrics 100:24-30

13. Aruoma OI, Halliwell B, Hoey BM, Butler J 1989 The antioxidant action of $\mathrm{N}$-acetylcysteine: its reaction with hydrogen peroxide, hydroxyl radical, superoxide, and hypochlorous acid. Free Radic Biol Med 6:593-597

14. Cotgreave I, Moldeus P, Schuppe I 1991 The metabolism of N-acetylcysteine by human endothelial cells. Biochem Pharmacol 42:13-16

15. Suter PM, Domenighetti G, Schaller M-D, Laverrière M-C, Ritz R, Perret C 1994 $\mathrm{N}$-acetylcysteine enhances recovery from acute lung injury in man. A randomized, double-blind, placebo-controlled clinical study. Chest 105:190-193

16. Bernard GR, Wheeler AP, Arons MM, Morris PE, Paz HL, Russell JA, Wright PE, The Antioxidant in ARDS Study Group 1997 A trial of antioxidants N-acetylcysteine and procysteine in ARDS. Chest 112:164-172

17. Domenighetti G, Suter PM, Schaller MD, Ritz R, Perret C 1997 Treatment with $\mathrm{N}$-acetylcysteine during acute respiratory distress syndrome: a randomized, doubleblind, placebo-controlled clinical study. J Crit Care 12:177-182

18. Ahola T, Lapatto R, Raivio KO, Selander B, Stigson L, Jonsson B, Jonsbo F, Esberg G, Stovring S, Kjartansson S, Stiris T, Lossius K, Virkola K, Fellman V 2003 $\mathrm{N}$-acetylcysteine does not prevent bronchopulmonary dysplasia in immature infants: a randomized controlled trial. J Pediatr 143:713-719

19. Roberts LJ, Morrow JD 2000 Measurement of F(2)-isoprostanes as an index of oxidative stress in vivo. Free Radic Biol Med 28:505-513

20. Goil S, Truog WE, Barnes C, Norberg M, Rezaiekhaligh M, Thibeault D 1998 Eight-epi-PGF $2 \alpha$ : a possible marker of lipid peroxidation in term infants with severe pulmonary disease. J Pediatr 132:349-351

21. Morrow JD, Frei B, Longmire AW, Gaziano JM, Lynch SM, Shyr Y, Strauss WE, Oates JA, Roberts LJ 2nd 1995 Increase in circulating products of lipid peroxidation (F2-isoprostanes) in smokers. Smoking as a cause of oxidative damage. N Engl J Med 332:1198-1203

22. Barden A, Beilin LJ, Ritchie J, Croft KD, Walters BN, Michael CA 1996 Plasma and urinary 8-iso-prostane as an indicator of lipid peroxidation in pre-eclampsia and normal pregnancy. Clin Sci 91:711-718

23. Montuschi P, Ciabattoni G, Paredi P, Pantelidis P, du Bois RM, Kharitonov SA, Barnes PJ 1998 8-Isoprostane as a biomarker of oxidative stress in interstitial lung diseases. Am J Respir Crit Care Med 158:1524-1527.

24. Collins CE, Quaggiotto P, Wood L, O'Loughlin EV, Henry RL, Garg ML 1999 Elevated plasma levels of F2 alpha isoprostane in cystic fibrosis. Lipids 34:551-556

25. Carpenter CT, Price PV, Christman BW 1998 Exhaled breath condensate isoprostanes are elevated in patients with acute lung injury or ARDS. Chest 114:1653-1659

26. Janssen LJ 2001 Isoprostanes: an overview and putative roles in pulmonary pathophysiology. Am J Physiol Lung Cell Mol Physiol 280:L1067-L1082

27. Ogihara T, Okamoto R, Kim HS, Nagai A, Morinobu T, Moji H, Kamegai H, Hirano K, Ogihara H, Tamai H, Mino M 1996 New evidence for the involvement of oxygen radicals in triggering neonatal chronic lung disease. Pediatr Res 39:117-119

28. Ogihara T, Hirano K, Morinobu T, Kim HS, Hiroi M, Ogihara H, Tamai H 1999 Raised concentrations of aldehyde lipid peroxidation products in premature infants with chronic lung disease. Arch Dis Child Fetal Neonatal Ed 80:F21-F25 
29. Schock BC, Sweet DG, Halliday HL, Young IS, Ennis M 2001 Oxidative stress in lavage fluid of preterm infants at risk of chronic lung disease. Am J Physiol Lung Cell Mol Physiol 281:L1386-L1391

30. Nycyk JA, Drury JA, Cooke RW 1998 Breath pentane as a marker for lipid peroxidation and adverse outcome in preterm infants. Arch Dis Child Fetal Neonata Ed 79:F67-F69

31. Winterbourn CC, Chan T, Buss IH, Inder TE, Mogridge N, Darlow BA 2000 Protein carbonyls and lipid peroxidation products as oxidation markers in preterm infant plasma: associations with chronic lung disease and retinopathy and effects of selenium supplementation. Pediatr Res 48:84-90

32. Yeo HC, Helbock HJ, Chyu DW, Ames BN 1994 Assay of malondialdehyde in biological fluids by gas chromatography-mass spectrometry. Anal Biochem 220:391-396
33. Volpe JJ 2003 Cerebral white matter injury of the premature infant-more common than you think. Pediatrics 112:176-180

34. Bagenholm R, Nilsson UA, Kjellmer I 1997 Formation of free radicals in hypoxic ischemic brain damage in the neonatal rat, assessed by an endogenous spin trap and lipid peroxidation. Brain Res 773:132-138

35. Bayir H, Kagan VE, Tyurina YY, Tyurin V, Ruppel RA, Adelson PD, Graham SH, Janesko K, Clark RS, Kochanek PM 2002 Assessment of antioxidant reserves and oxidative stress in cerebrospinal fluid after severe traumatic brain injury in infants and children. Pediatr Res 51:571-578

36. Inder T, Mocatta T, Darlow B, Spencer C, Volpe JJ, Winterbourn C 2002 Elevated free radical products in the cerebrospinal fluid of VLBW infants with cerebral white matter injury. Pediatr Res 52:213-218 